\title{
El aprovechamiento de madera teosa de Pinus halepensis Mill. y los hornos de alquitrán en Ibiza (Islas Baleares)
}

Pinus halepensis Mill. resin exploitation and pitch kilns on Ibiza (Balearic Islands, Spain)

\author{
Ribas, V.A. ${ }^{1}$; Dopazo, C.; ${ }^{* 2}$ \\ ${ }^{1}$ Escuela Técnica Superior de Ingeniería Agronómica y del Medio Natural. \\ Universitat Politècnica de València. Camino de Vera s/n. 46022 Valencia \\ ${ }^{2}$ Instituto Universitario de Ingeniería del Agua y del Medio Ambiente (IIAMA). \\ Grupo de Ciencia y Tecnología Forestal. Universitat Politècnica de València. \\ Camino de Vera s/n. 46022 Valencia
}

"Autor para correspondencia: cardogon@agf.upv.es 


\title{
Resumen
}

Por sus características particulares, el aprovechamiento de resina en la isla de Ibiza se ha realizado hasta hace unas décadas siguiendo procedimientos arcaicos, basados en el tratamiento del árbol para la producción de madera enteada, la posterior extracción de esta madera y su procesado mediante destilación por combustión en hornos primitivos. Se trata de actividades ya abandonadas a lo largo del siglo XIX en la península ibérica, en parte desconocidas y sin apenas información publicada, como es el caso del tratamiento para la producción y aprovechamiento de la madera enteada. El objetivo es aportar nuevos conocimientos y contribuir a la descripción del proceso tradicional del aprovechamiento de productos resinosos de Pinus halepensis Mill. en la forma realizada en la isla de Ibiza, incluida la preparación del árbol para la producción de madera enteada, su extracción y el posterior procesado con técnicas rudimentarias de destilación de la madera en hornos de alquitrán. A partir de la recogida sobre el terreno de información oral, correspondiente a personas que han realizado la actividad o han tenido un contacto directo con antiguos trabajadores del sector, así como del reconocimiento de materiales e infraestructuras empleados, se aportan nuevos datos de los procedimientos empleados, así como de las características de los árboles y hornos utilizados. Se describe el tratamiento, el aprovechamiento de la madera enteada y el procesado de los productos resinosos hasta su venta tras la primera transformación. Se amplían las localizaciones de hornos existentes con dos nuevos hornos. Se discuten los posibles motivos del mantenimiento de esta actividad hasta hace pocas décadas.

Palabras clave: encrità, pega, peguera, pez, resina, tea

\begin{abstract}
Due to Ibiza's singular characteristics, the exploitation of resin had been maintained until some decades ago in an archaic way, based on the treatment of the tree as a producer of fatwood, which was extracted and processed through slow, controlled combustion in primitive pitch kilns. In contrast, these activities were abandoned during the XIX century in the iberian peninsula, becoming partly unknown and little represented in the literature. The objective of this article is to contribute to the understanding of this ancient industry both with new information and knowledge and explaining the process required in traditional Pinus halepensis Mill. pitch fabrication. Tar production on Ibiza, including the tree preparation, fatwood extraction and its combustion as well as rudimentary distillation process in tar ovens has been studied by interviewing people who have worked in this sector or people who have been directly involved with former workers. Materials and infrastructures used have been examined and provide new data about the trees' and ovens' characteristics, as well as about the techniques traditionally used. The treatment of the tree is described, and so it is the extraction and uses of pine tar. Finally, two previously non-cited ovens are identified and located. The possible reasons for the late disappearance of these practices are also discussed.
\end{abstract}

Keywords: fatwood, oven of pitch, pitch, tar. 


\section{Introducción}

La isla de Ibiza ha estado en la ruta de grandes civilizaciones, que en los diferentes periodos han explotado y aprovechado los recursos disponibles. La pesca, la agricultura o las salinas han sido claves en su desarrollo, ya fuera para consumo local o para el comercio. De entre todos los sectores de trabajo hay uno que ha tenido hasta hace pocas décadas una importancia crucial por ser transversal a todos los demás: el sector forestal. Elementos como la madera para la construcción, la leña como fuente de energía y los alimentos para las personas y el ganado son una pequeña muestra de todo el potencial de oportunidades y materiales que el bosque ibicenco ofrecía a sus gentes. Uno de los aprovechamientos forestales relevantes ha sido el de productos resinosos procedentes de Pinus halepensis Mill., la principal especie de pino presente en la isla.

La producción de pez en Ibiza, su empleo para múltiples aplicaciones e incluso la exportación se constata por escrito desde la época romana y se mantiene durante siglos (Gil et al., 2002). Existen referencias documentadas del aprovechamiento de productos resinosos de finales del siglo XIII y siglo XIV (Guerau, 1973; Gil et al., 2002) en las que se hace mención a la prohibición de extracción de pez y alquitrán de la isla, o del permiso para la exportación a Mallorca y otras tierras del Reino de Aragón de madera, pez, tea o resina. También se citan nuevas prohibiciones de exportación de alquitrán y pez en el siglo XVIII, frente a la escasez local de los productos y para intentar evitar la sobreexplotación de los montes de la isla. Estas referencias permiten hacerse una idea de la importancia que el aprovechamiento de productos resinosos procedentes de Pinus halepensis ha tenido en la historia de la isla de Ibiza. Por una parte, desde un punto de vista de conservación del monte, por los graves daños que podía llegar a causar la sobreexplotación de las masas y los consiguientes efectos ambientales, sociales y económicos, tanto a corto como a largo plazo. Por otra parte, desde un punto de vista económico y social, por la relevancia que los productos resinosos adquirían, tanto a nivel comercial como para cubrir una serie de necesidades locales, ya fueran familiares o industriales. Entre estas últimas es muy relevante la necesidad de la pez para el calafateo de los barcos en una pujante industria de la construcción naval en las Islas Baleares durante varios siglos (Gil et al., 2002), así como para la impermeabilización de envases u otros útiles que servían para la producción y transporte de diversos recursos estratégicos para las islas.

En el marco de un trabajo realizado en la isla de Ibiza (Ribas, 2019) se ha observado la ausencia, en las décadas centrales del siglo XX, de referencias al empleo de técnicas de extracción de resina similares a las empleadas en aquellos momentos en la mayor parte de la península ibérica, con la práctica de heridas longitudinales en el fuste del pino (picas) para fomentar la secreción de la resina y recogerla en un recipiente (pote). Por el contrario, han sido frecuentes las referencias al mantenimiento en la isla, al inicio de ese mismo periodo, de otros procedimientos de extracción y procesado más antiguos, que no solo buscaban la resina secretada por el árbol, sino también el fomento de la producción y la extracción de madera teosa (Real Academia Española, 2019) o enteada. Esta madera se caracteriza por una im- 
pregnación con resina de los elementos traqueales del xilema secundario, que da lugar a un contenido de resina en la madera anormalmente alto (S.E.C.F., s.f.). La madera posteriormente era extraída y procesada con técnicas rudimentarias de destilación, basadas en la quema de la madera en unos hornos especiales denominados en la isla forns d'encrità o pega (hornos de alquitrán). La información existente sobre esta forma de producción y extracción de madera teosa de árboles vivos es muy escasa, siendo una actividad en gran parte desconocida. Parece ser una actividad realizada desde la antigüedad, que había sido abandonada bastantes décadas antes en otras áreas de la Península ibérica (Fàbrega, 2006; Orduna, 2014). Los daños producidos al árbol, la fuerte demanda de madera para otros usos y la introducción de métodos más eficaces para la extracción de la resina y su procesado industrial supusieron el final de la misma a lo largo del siglo XIX. Se mantuvieron en el tiempo procesos similares en cuanto al proceso de destilación de madera teosa y otros restos con resina, para la obtención de pez negra a partir de estos residuos, pero ya ligados a algunas fábricas de resina o de forma residual. (Iturralde y Elorrieta, 1914; Prados, ca. 198-; Fàbrega, 2006; Michavila, 2010; Orduna, 2014). En el caso de la isla de Ibiza, los aprovechamientos basados no sólo en el procesado de teas, sino también en su producción y aprovechamiento, parecen haber perdurado en formas tradicionales, arcaicas, durante un periodo de tiempo más prolongado que en otros puntos de la península ibérica, muy posiblemente debido a sus particularidades, ligadas a su carácter insular y a la presencia de Pinus halepensis como única especie del género bien representada. Este hecho permite la recogida de testimonios orales más próximos en el tiempo y la recopilación de nueva información respecto a una actividad que, a pesar de haber sido relevante durante muchos siglos en sus formas arcaicas, es en parte desconocida.

Por otra parte, el abandono total de esta actividad en la isla, en torno a los años 50 y 60 del siglo pasado, se produce en el mismo momento en que se inicia un cambio social y económico brusco en la isla, con el abandono del sector primario y el establecimiento de una potente industria turística. Como consecuencia de los cambios producidos y del paso del tiempo, tanto las tradiciones como ciertos conocimientos asociados al uso de los campos y los montes se van perdiendo. En este contexto de pérdida de acervo cultural ligado a la vida rural, la recuperación del conocimiento sobre uno de los aprovechamientos forestales relevantes en la isla desde hace muchos siglos tiene un interés cultural, de recuperación de conocimientos etnográficos presentes todavía en la tradición oral. A su vez, estos conocimientos también pueden servir como complemento a una pujante actividad turística que contemple el potencial de las actividades tradicionales rurales ibicencas para favorecer un desarrollo rural y turístico más sostenible en la isla. La recuperación de esta actividad con carácter puntual puede tener un interés cultural y de turismo con base etnográfica.

\section{Objetivos}

El objetivo de este artículo es aportar nuevos conocimientos y contribuir a la descripción del proceso tradicional del aprovechamiento de productos resinosos de 
Pinus halepensis en la forma tradicional realizada en la isla de Ibiza, desde la preparación del árbol hasta la puesta en el mercado de la pez o alquitrán. Incluirá la producción de madera enteada, su extracción y el posterior procesado con técnicas rudimentarias de destilación seca en hornos de alquitrán (forns d'encrità o pega).

\section{Materiales y métodos}

El estudio se circunscribe a la isla de Ibiza (Islas Baleares). Se ha realizado una búsqueda y revisión de la bibliografía existente sobre el tema y entre los meses de agosto de 2018 y agosto de 2019 se ha recopilado información oral sobre el terreno. La posibilidad de obtener información directa de personas que hubieran realizado el aprovechamiento antes de su abandono ha sido muy limitada, debido al tiempo transcurrido, y ha quedado circunscrita a una persona: Joan Serra Bonet. Sin embargo, sí ha sido posible entrevistar a personas que han tenido un contacto directo con antiguos trabajadores del sector y que han podido recoger y ahora transmitir información oral de personas ligadas a la actividad. Es el caso de Vicent Marí Serra "Palermet", quien ha conocido el oficio a través de su abuelo, peguero, y que se ha dedicado a la recopilación etnográfica y a la restauración de hornos; de Antoni Boned Boned "Basora", quien ha recogido testimonios y ha construido un horno para la recuperación de esta actividad; de Antoni Tur Riera "Sendic", mestre d'aixa conocedor de todas las técnicas de construcción naval tradicional de Ibiza y de María Ribas Noguera de "Can Gibert" quien, entre otras actividades, dedicó parte de su vida a la recolección manual de resina. A partir de estas entrevistas también se han situado nuevas localizaciones de antiguos hornos de alquitrán.

\section{Resultados}

Las informaciones orales aportadas permiten describir el aprovechamiento de los productos resinosos del pino, los procesos de transformación para la fabricación del alquitrán y sus usos tradicionales de la siguiente forma:

\subsection{El aprovechamiento y procesado de los productos resinosos}

\subsubsection{La selección de los pinos y su preparación previa}

Los pinos a aprovechar eran seleccionados en base a unos criterios básicos. El primero y más importante era el diámetro. A partir de aproximadamente $20 \mathrm{~cm}$ de diámetro normal (diámetro a 1,30 $\mathrm{m}$ de altura) el pino era considerado susceptible de ser empleado para este fin, pero se buscaban los pies más gruesos, para los que se presuponía una mayor cantidad de resina a extraer (Boned, 2018; Marí, 2019). Otras características como el porte o la poda natural también podían ser importantes. Los informadores coinciden en que, en términos generales, se buscaban pinos que no tuvieran un porte excesivamente achaparrado y con aproximadamente entre 


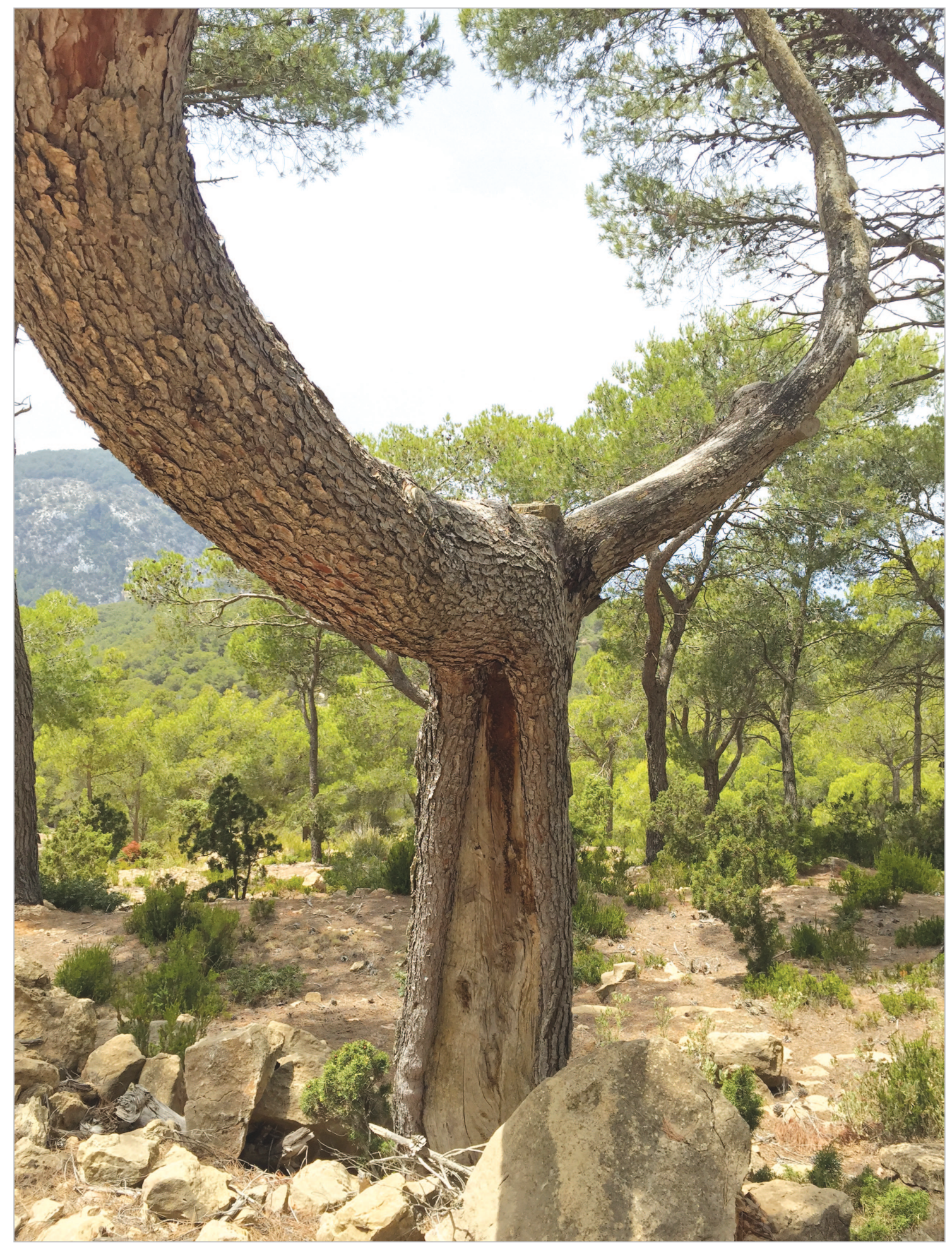

Figura 1. Pino de la Serra des Camp Vell del que se aprovecharon teas hasta hace aproximadamente 80 años. Se observa el corte de la parte superior de la copa (escimerolar es pi) y el área preparada (parar es $p i)$ en la que se extrajeron las teas. Se aprecia que una parte de la gran herida se ha ido cerrando con el paso del tiempo. 
uno y dos metros libres de ramas en la base del fuste. En general parece ser que eran preferidos los pinos que estuvieran cerca de vías de comunicación, muy posiblemente por la mayor facilidad de acceso y saca del producto.

Al árbol se le cortaba la parte superior de la copa (Boned, 2018; Marí, 2019). Esta mutilación, conocida como escimerolar es pins, se realizaba con la intención de aumentar el crecimiento de la madera de las partes basales del fuste y para concentrar la resina producida por el árbol en dicha zona (Fig. 1). Según testimonios recogidos por Boned (2018), el corte nunca debía eliminar más de un tercio de la altura de la copa. Si la parte del tronco destinada al aprovechamiento no estaba desprovista de ramas se procedía a su poda en ese momento. Una vez escimerolat el pino se procedía a lo que se denominaba parar es pi (Fig. 2). La operación de parar es pi consistía en hacer una herida al fuste del pino, quitando la corteza y eliminando con ella floema y cambium. Se llegaba así al leño o madera, pero sin afectar a éste (Fig. 3). Boned (2019) indica que la extracción se hacía en la cara sur o suroeste del fuste, orientación también empleada en otras localizaciones por indicar los resineros que se produce una mayor cantidad de resina. Las dimensiones iniciales (Serra, 2019) se situarían en torno a unos $25 \mathrm{~cm}$ de anchura, no siendo recomendable, tras sucesivas operaciones de extracción, que la herida superara la mitad de la circunferencia de la sección (Fig. 4). La altura era variable y limitada a aquella que se podía trabajar con el hacha desde el suelo. Marí (2018) hace referencia al uso ocasional de maderas que, apoyadas en el árbol, servían para que subido a ellas el peguero pudiera incrementar la altura de trabajo en el tronco unos 30 ó $40 \mathrm{~cm}$. Esta fase del proceso se solía realizar en primavera o durante el inicio del verano, periodo con una mayor actividad vegetativa y de producción de resina del árbol.

\subsubsection{La extracción de "ses teies": la madera enteada}

Pasados dos años desde que se parava el pino se procedía a la extracción de la madera teosa que se había formado. El procedimiento requería de gran precisión y práctica: el hombre, con un hacha como única herramienta, se colocaba de forma que el recorrido de ésta estuviera contenido en un plano tangente a la sección del pino. De esta forma con movimientos de arriba hacia abajo iba sacando astillas de madera enteada. El tamaño de estas astillas era función de la destreza del que las sacaba, siendo mejor cuanto más largas (Marí, 2018). El hacha debía ser de mango largo y hoja larga y estrecha, muy afilada (Boned, 2018). La profundidad que alcanzaba esta impregnación en la madera era variable y solía alcanzar entre 1 y 3 ó $4 \mathrm{~cm}$ de profundidad (Boned, 2018; Marí, 2018).

Una vez extraídas las astillas de tea se dejaban en el pie del pino para que recogieran la secreción de resina que resbalaba por el corte. También se podía dejar un pequeño recipiente $u$ hoyo en la base con este fin y recoger la resina que escurría por el tronco.

El procedimiento se repetía cada dos años durante la vida del árbol (que veía acortada su duración). Marí (2018) y Boned (2018) hacen referencia al empleo de "matas" alternantes, de forma que un año se extraían las teas del grupo de árboles que se trabajaba por el equipo (mata) y al año siguiente se extraían de otro grupo de 


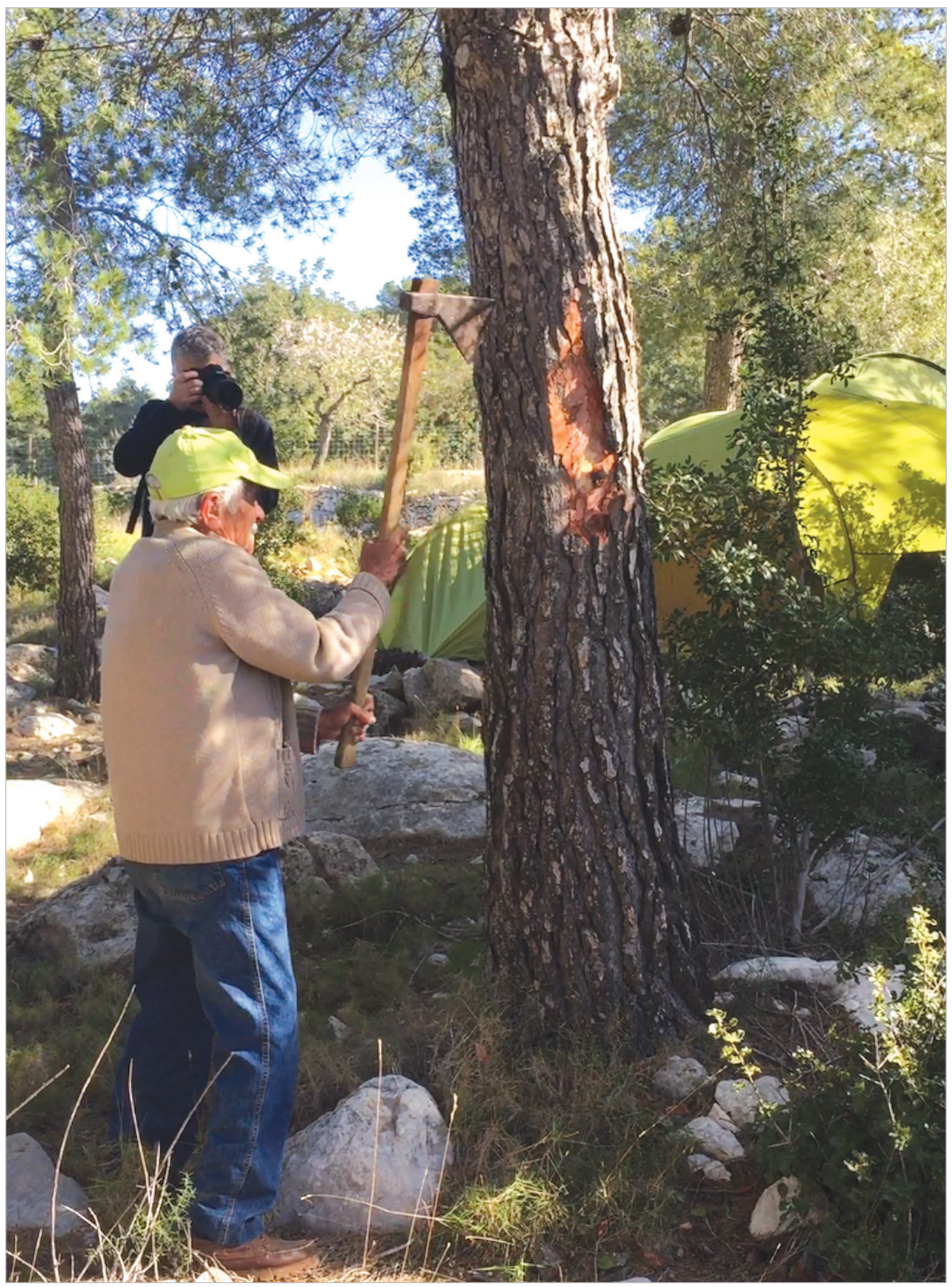

Figura 2. Demostración de la preparación del pino (parar es pi) por Joan Serra Bonet, que en su juventud llegó a ver y aprender este oficio de su padre y tío. En este caso no se ha procedido a la corta previa de la parte superior de la copa (escimerolar es pi). 


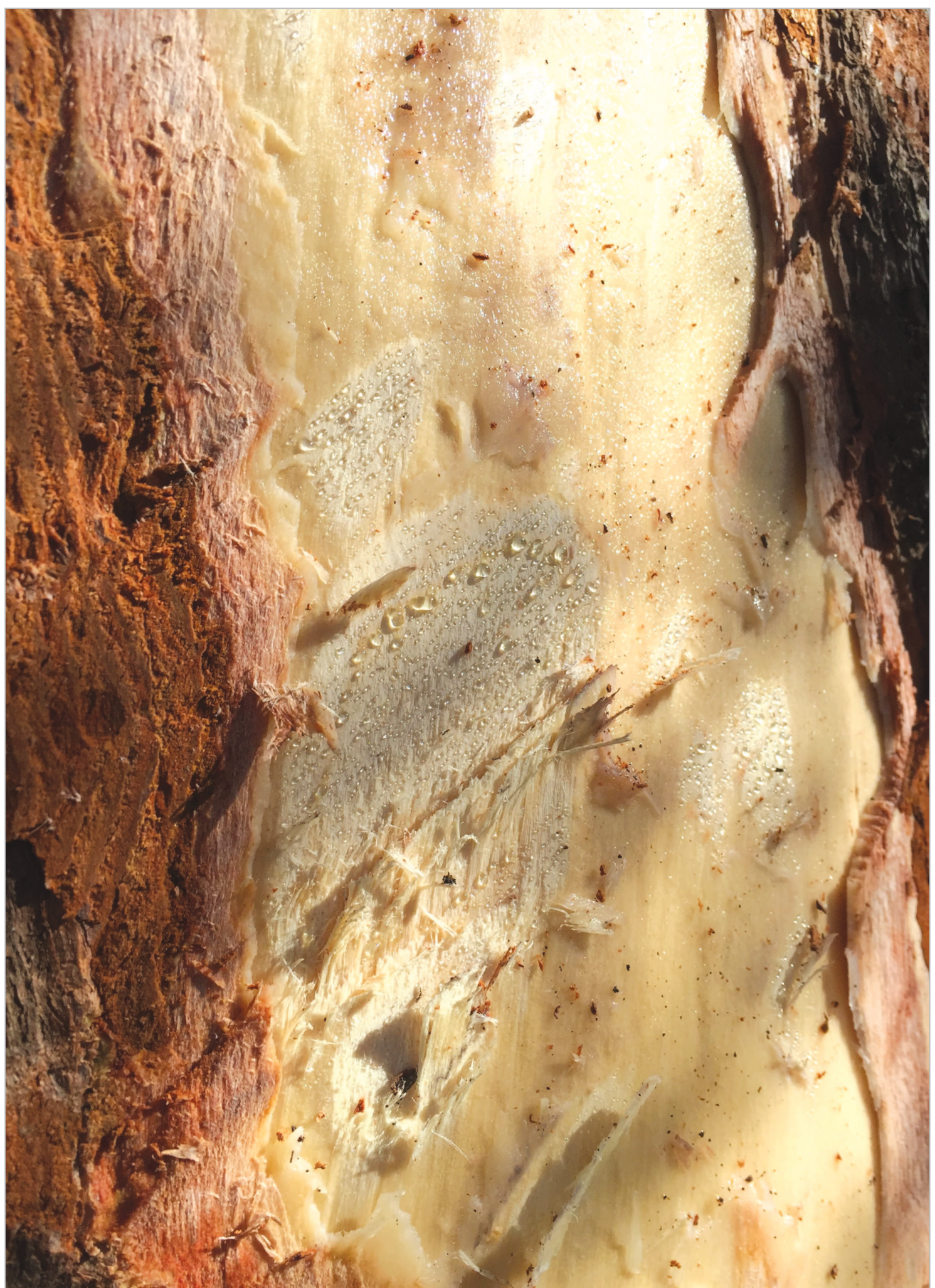

Figura 3. Herida recién abierta en un pino. Detalle de las capas afectadas (de izquierda a derecha): corteza, floema, cambium y xilema. 


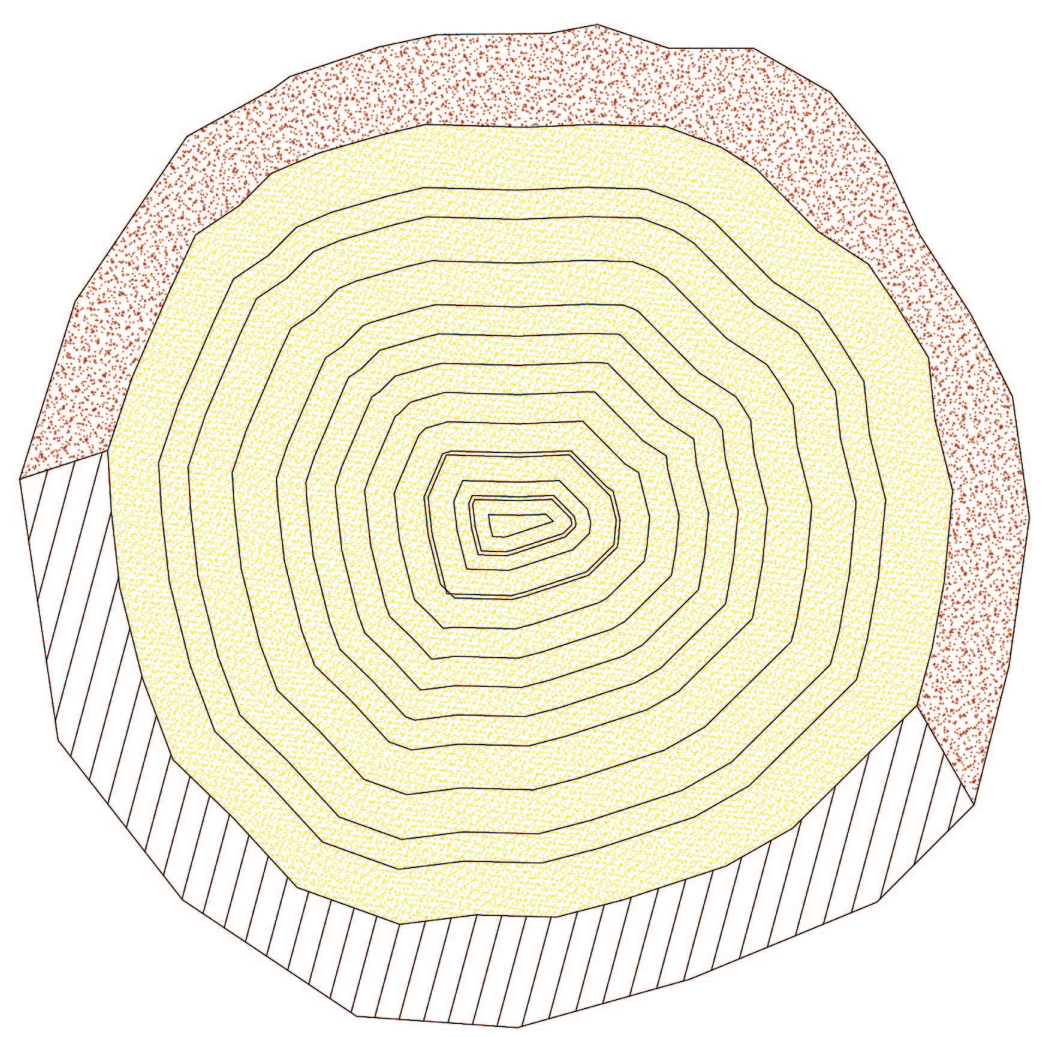

Figura 4. Croquis de la sección transversal del fuste de un pino preparado (pi parat). La zona rayada representa la parte que como máximo y a lo largo de diferentes turnos, es recomendable que sea afectada por la extracción de teas.

Fuente: elaboración propia a partir de diversos testimonios.

árboles y se dejaba descansar al primero, alternándose los grupos cada año. Serra (2019) también indica que los ha realizado con turnos de 2 años. Marí (2019) hace referencia a turnos de hasta 5 años en periodos anteriores, de menor intensificación.

Al estar el cambium activo en el resto de la sección, el pino seguía engrosando, pero cada vez con menor vigor, especialmente en el lateral del árbol afectado por la extracción. Según Boned (2018), a medida que se sucedían las extracciones, la tea procedente de la madera central del tronco era de menor calidad, hecho que puede ser debido a la llegada al duramen, una zona con canales resiníferos que han dejado de ser funcionales, en la que la impregnación de resina tras las heridas es menor (Fig. 5).

\subsubsection{Los hornos de alquitrán}

En la isla de Ibiza existían una serie de hornos especiales en los que se procedía a la extracción de la resina contenida en las teas que se astillaban del pino: los hornos de alquitrán, o en ibicenco forns d'encrità (Fig. 6). Es una construcción formada por dos partes diferenciadas: el horno propiamente dicho (es forn), consisten- 


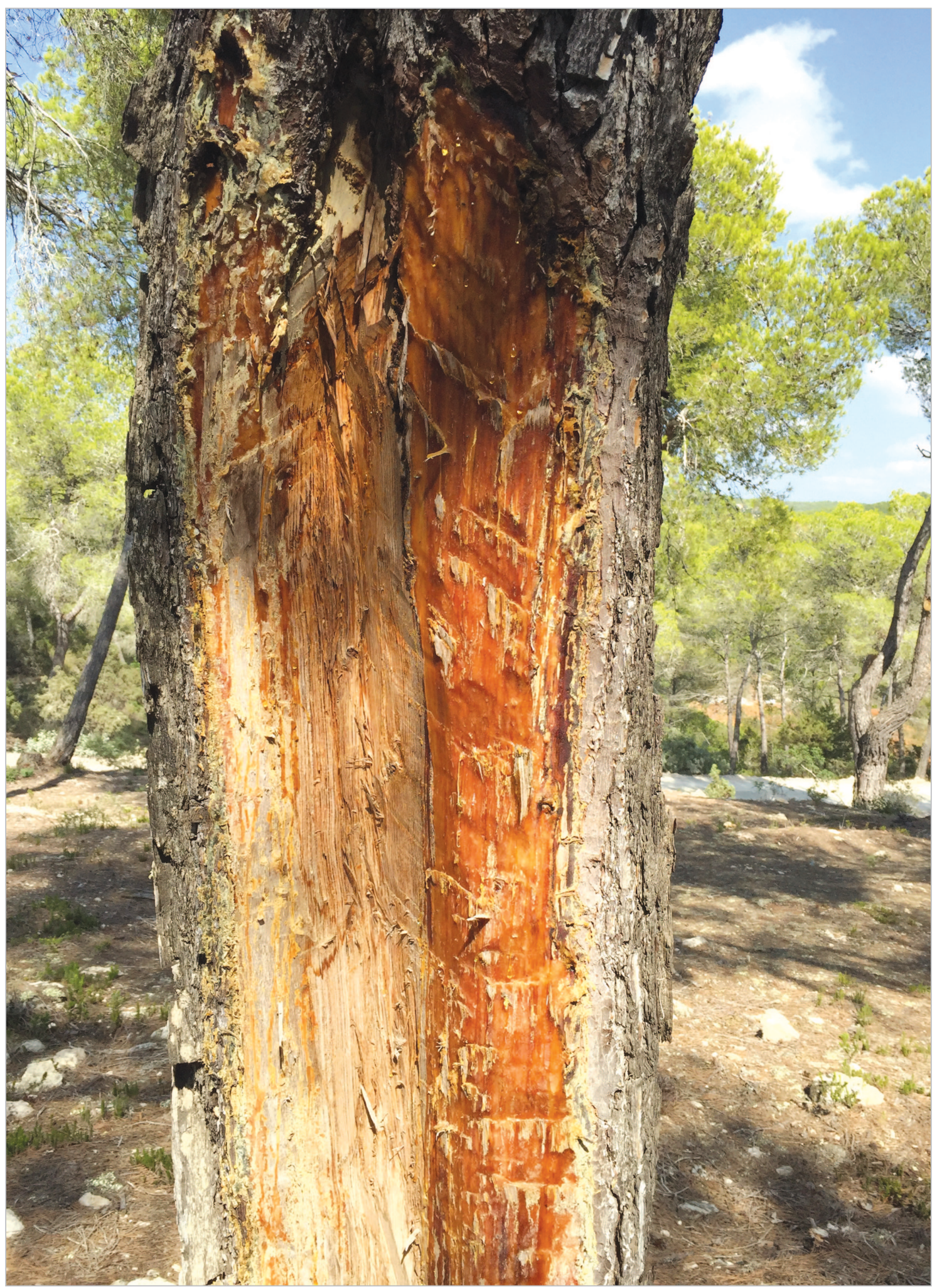

Figura 5. Pino donde se han extraído teas (hace un año) en una antigua cara resinada. Se aprecia la diferente impregnación de la madera en el duramen (izquierda) y en la albura (derecha). 
te en una gran caverna abovedada de planta ovoidal en la que se colocan las teas, y un depósito cilíndrico exterior ( $s$ 'olla) situado en la base del horno. Ambas partes se unen con un conducto llamado rajador, que atraviesa el horno y desembocaba en $s$ 'olla en un agujero visible desde el exterior, que recibe el nombre de es forat. El horno tiene otra abertura superior, ligeramente ladeada respecto al eje central y denominada boca, por donde se llena con las astillas de madera enteada. Todas las informaciones recogidas hacen referencia a la construcción de los hornos apoyada en un desnivel del terreno. La parte posterior de la cavidad mayor (es forn) se apoyaba en el desnivel y facilitaba su carga por la parte elevada del terreno a través de la boca superior. La olla era excavada en la parte inferior. En Guerau (1973) aparecen representados una planta y una sección tipo de un horno de alquitrán. Marí (2018) hace referencia a la existencia de dos tipos de horno en la isla. En el suroeste abundaban unos hornos más pequeños, de carácter más familiar. Se podían llenar con astillas de tea procedentes de aproximadamente 25 a 30 pinos (aproximadamente 1,5 $\mathrm{m}^{3}$ de volumen de forn), que se caracterizaban por tener la parte superior plana, con una abertura en general mayor. En el norte y noreste de Ibiza abundaban otro tipo de hornos, más grandes y de carácter más industrial, que daba cabida a astillas de 80 pinos o más ( $2 \mathrm{~m}^{3}$ o más). Hay también algunos hornos de un tamaño intermedio entre ambos.

Marí (2018) hace referencia al tradicional empleo de la denominada pedra morta, (piedra caliza de origen sedimentario del Cuaternario). El mortero para las juntas era tierra arcillosa que al encender el horno por primera vez se convertía en cerámica (lo que da idea de que en él se llegaban a temperaturas cercanas a $400^{\circ} \mathrm{C}$ ).

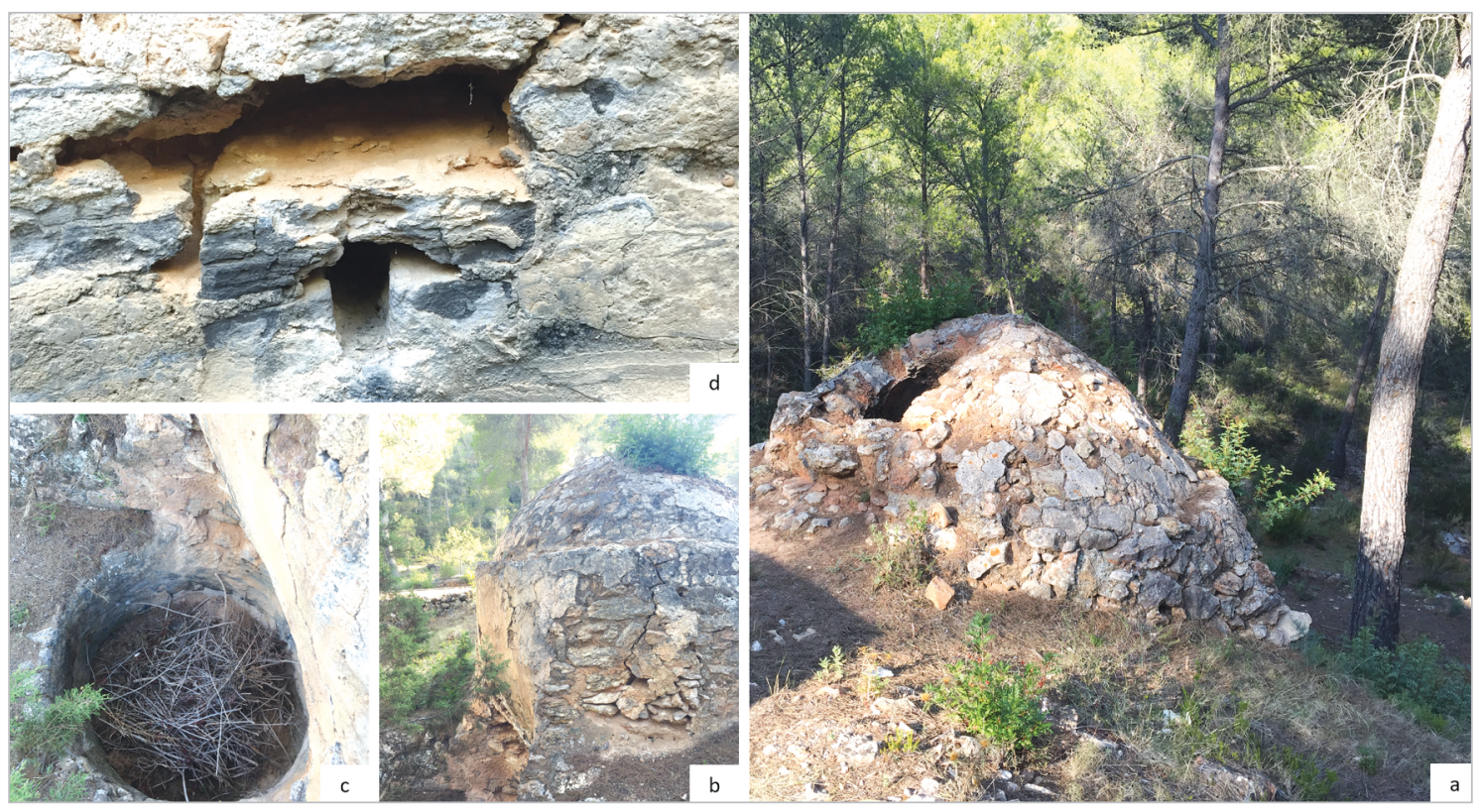

Figura 6. Horno de alquitrán de Ses Marrades de Corona (Santa Agnés de Corona): a) vista posterior con la boca parcialmente derruida; b) vista lateral en la que se aprecia s'olla en la parte inferior; c) detalle de s'olla; d) detalle de la salida del rajador a s'olla (es forat), ennegrecido por el goteo del alquitrán. 
En ocasiones podía hacerse un recubrimiento externo con cal viva, que aumentaba la duración y la resistencia de la construcción.

\subsubsection{E1 funcionamiento de los hornos de alquitrán}

Las teas extraídas de los pinos y apiladas en su pie eran recogidas y llevadas al horno. Allí eran colocadas en el interior de la cavidad mayor (es forn) de manera vertical y con la mayor densidad posible para no perder rendimiento en el horno. En la parte basal del interior del horno, en el lugar donde se inicia el conducto que lo atraviesa para ir a parar a la olla, se dejaba un pequeño hueco o cavidad construido con las propias teas (Boned, 2018), aunque también era común la colocación de una pequeña losa para cumplir con esta función (Marí, 2018).

A continuación se sellaba la olla. Se solía hacer colocando un techo formado por troncos de pino (de aproximadamente 10 a $15 \mathrm{~cm}$ de diámetro) entrecruzados formando una malla, seguido de una capa de 20 a $30 \mathrm{~cm}$ de espesor de ramas más finas para acabar con aproximadamente un metro de tierra. El objetivo era conservar el alquitrán una vez que éste se acumulaba en este depósito, evitando la pérdida de la parte volátil y de sus propiedades (que $s$ 'embravàs), así como una combustión no deseada en la olla por la entrada de aire (Boned, 2018). Marí (2018) y Boned (2018) hacen referencia, una vez lleno y sellado el horno, a su encendido por la parte superior, mediante una hoguera. En el caso de los hornos más grandes era común el empleo de una piedra plana en la parte superior del horno, que permitía el tapado de la boca al ser desplazada mediante una vara larga de pino o sabina. En caso de que el fuego se volviera demasiado agresivo (la madera enteada tiene una alta inflamabilidad y alto poder calorífico), el tapado de la boca permitía controlar la entrada de aire al horno y controlar la intensidad del fuego.

El alquitrán se obtenía mediante la destilación seca de la madera teosa. Cuando se encendía el horno, las teas superiores exudaban la resina que tenían en su interior por acción del calor. Esta sustancia empezaba a gotear e iniciaba un viaje hacia el fondo del horno, donde se concentraba y, atravesando el rajador, entraba al depósito exterior ( $s$ 'olla). La exudación de resina de las teas debía producirse con el calor producido y antes de que éstas entraran en ignición. Para ello se precisaba una alta temperatura, pero sin un fuego intenso, con el objeto de evitar en lo posible la combustión de la resina y evitar así la disminución de rendimiento del horno. Una vez apagado el horno, el residuo que quedaba dentro se reducía a cenizas y algún carbón. La información relativa a la duración del proceso es variada. Marí (2019) hace mención del encendido del horno por la tarde y su combustión total a la mañana siguiente. Boned (2018) hace referencia a experiencias recientes en las que en 4 a 5 horas en un horno de aproximadamente $0,5 \mathrm{~m} 3$ el proceso estaba completamente finalizado. Era común cocer un segundo horno al vaciar los restos de la primera hornada, para aprovechar que estaba caliente y reducir la duración de la segunda hornada.

Se debían esperar aproximadamente dos días para destapar el depósito de la olla dónde estaba el alquitrán, con el propósito de dejar enfriar la mezcla. Lo que se encontraba en él era una capa de sustancia parda negruzca, semisólida en la parte su- 
perior y densa en la parte inferior (Fig. 7). También era común que debajo de esta capa de encrità se presentara una pequeña capa de agua como residuo (el agua, al ser más densa, descendía al fondo de la olla).

Marí (2019) hace referencia a la posibilidad de obtención de pega (pez) a partir de la quema del alquitrán. Tanto Marí (2019) como Boned (2018) hacer referencia a una segunda acepción según la cual también se denominaba pega al alquitrán viejo.

\subsection{El alquitrán: usos y aplicaciones}

Cuando se destapaba el depósito de la olla, se realizaba la venta a los vecinos, ya fuera a pie de horno, de forma ambulante o a través de tiendas locales donde la depositaban los horneros, como Can Belloteta en Sant Jordi. Era común que los ve-

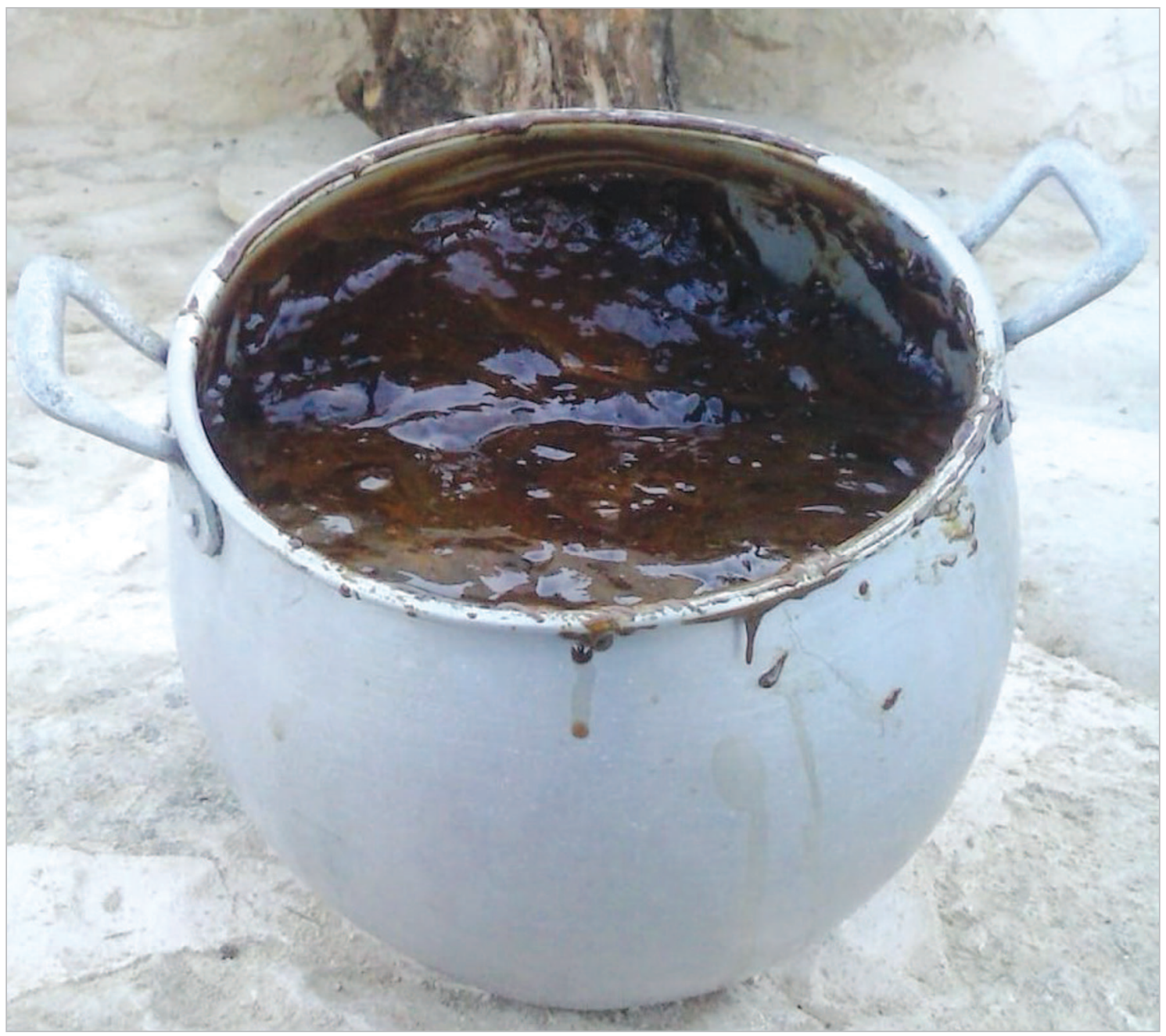

Figura 7. Detalle de un recipiente lleno de alquitrán de Pinus halepensis elaborado en una hornada de prueba realizada en febrero de 2019. 
cinos realizaran la compra aportando un recipiente ya destinado en sus casas payesas para el alquitrán, que era un producto crucial en aquellos tiempos. Se solía comprar con dinero, aunque también se daban acuerdos de trueque, intercambiando el alquitrán por otro producto aportado por el comprador (Cardona, 2019; Marí, 2019).

Los usos eran muy diversos. Uno de los principales era el de aumentar la resistencia de las zapatillas tradicionales hechas en la isla, las espardenyes. Este tipo de calzado, fabricado con esparto (Stipa tenacissima L.) y pita (Agave americana L.), necesitaba de una sustancia que les diera resistencia. Para ello, la gente de la isla solía aplicarles sucesivas capas de alquitrán (encritanar ses espardenyes). Se calentaba para hacerlo fluido y, siempre cerca de una fuente de calor, se untaba toda la suela con encrità repetidas veces. Por sus cualidades impermeables, era común su aplicación en materiales que precisaran de esta característica. Boned (2018) pone como ejemplo su empleo en partes de las norias y molinos de extracción de agua, partes de vigas que tuvieran que ir empotradas a la pared o al suelo y ruedas de carros. Marí (2019) hace referencia a la aplicación en la vestimenta y herramientas de los hombres que trabajaban en las salinas (era típico que los sombreros donde apoyaban las cestas cargadas de sal o sus prendas de trabajo estuvieran alquitranadas, así como los cubos de esparto utilizados). En uno de sus principales usos, calafatear las embarcaciones, Tur (2018) hace referencia a su empleo en los astilleros primitivos, donde los constructores (mestres d'aixa) de embarcaciones tradicionales ibicencas (llamadas llaüts i xalanes), empleaban alquitrán y pez para pintar y untar tanto las partes que tenían que tener un contacto directo con el agua como las que simplemente iban a estar en contacto con la humedad. Aplicaban estos productos derivados de la resina a quillas, interiores, juntas e incluso cabos y velas mediante alquitrán, estopa, sebo y aceite. Marí (2018) y Boned (2018) hacen referencia a otros usos muy diferentes: hervido y mezclado con aceite, lo aplicaban a las heridas de los animales para prevenir infecciones y necrosis; también era común su empleo combinado con varas de madera para inmovilizar articulaciones rotas en el ganado. Con la entrada de otros productos sustitutivos, como por ejemplo la innovadora suela de neumático para zapatos hace aproximadamente 80 años, la producción de alquitrán disminuyó drásticamente.

\subsection{La distribución de los hornos de alquitrán en la isla de Ibiza}

En la Figura 8 se recoge la distribución de los 18 hornos de alquitrán en buen estado localizados en la isla de Ibiza por Guerau (1973) junto con la de dos nuevos hornos (Fig. 9) localizados tras los testimonios orales (Bonet, 2018; Marí, 2018). Se aprecia en la figura que la mayor parte de los hornos se concentran en las zonas más forestales de la isla, que se corresponden con Sant Josep de Sa Talaia, Santa Agnès de Corona y Sant Joan de Labritja. Marí (2018) hace referencia a nueve nuevos hornos adicionales en el municipio de Sant Josep de Sa Talaia, localizados en las fincas de Cas Serres, Cas Costes, Ca Na Vergera, Es Fornet, Can Musón, Can Xico Serra, Es Torrentàs, Es Forn Figuera y Can Gustí. 


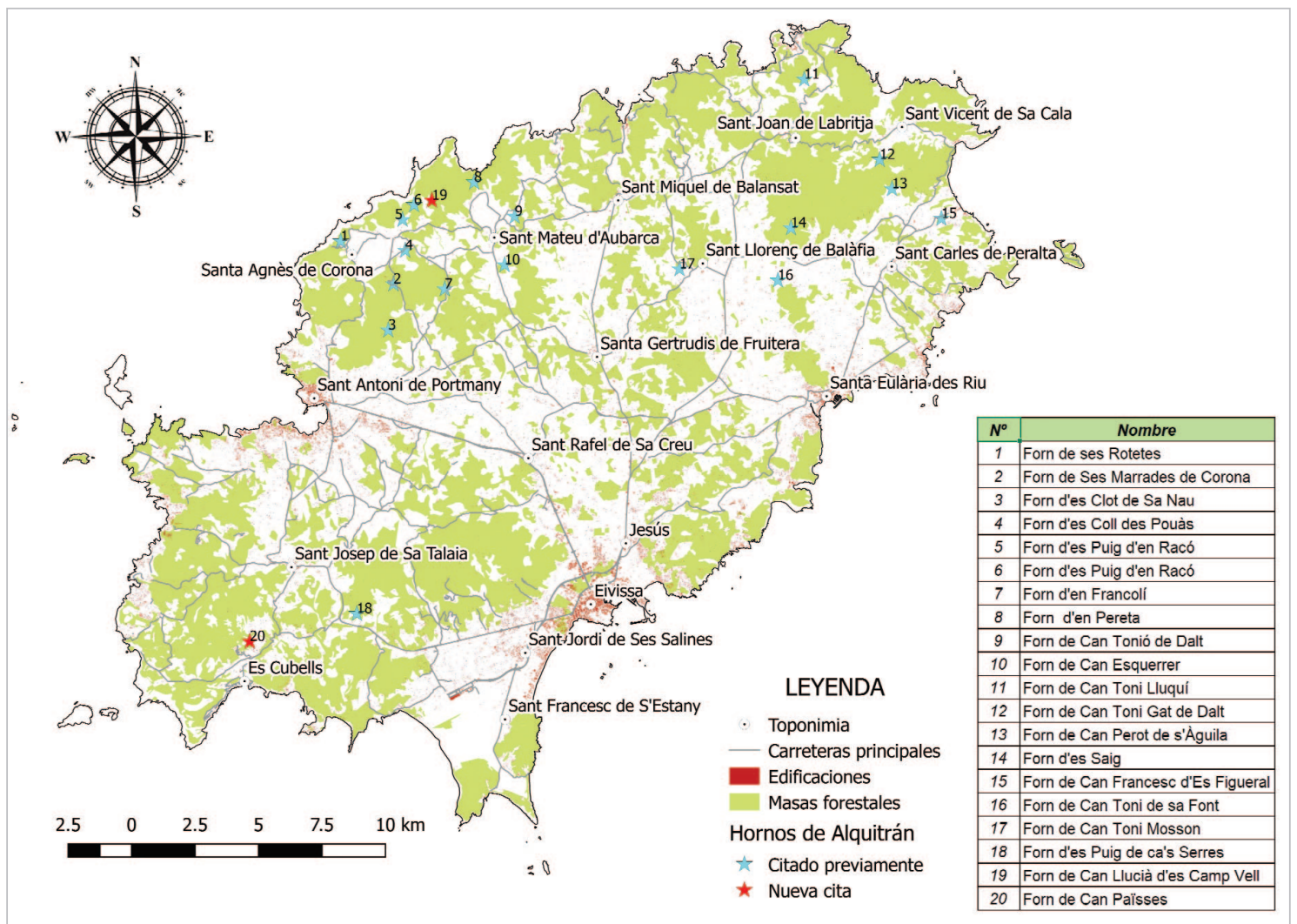

Figura 8. Localización de los hornos de alquitrán citados en la isla de Ibiza.

Fuente: elaboración propia a partir de Guerau (1973) e información facilitada por Boned (2018).

Estos hornos antaño fueron numerosos y solían estar combinados con otras formas de aprovechamiento como hornos de cal o sitges (llamadas así en ibicenco las carboneras), conformando unas primigenias industrias forestales. Sin embargo, si bien los hornos de cal o las carboneras duraron mucho más tiempo, esta forma de aprovechamiento de la resina del pino carrasco se dejó de realizar hace muchos decenios. Boned (2018) y Marí (2018) hacen referencia al cese de su actividad en torno a la década de los años treinta del siglo XX. Bonet (2018) hace referencia al uso del horno de Can Llucià para producción familiar hasta aproximadamente 1960.

\section{Discusión}

No se ha recogido ninguna información relativa a la existencia de técnicas para la extracción de resina en árboles vivos, pero sí de la recogida de las secreciones de resina existentes de forma natural. María Ribas (2018), de Can Gibert, hace referencia a haber recogido secreciones de resina que los árboles generaban de forma natural por diferentes heridas (rozaduras de ganado, roturas de ramas o cortas). Indica que era una actividad realizada por mujeres y niños para la posterior venta del producto; era común la venta de esta resina, a seis pesetas el kilogramo, a comer- 


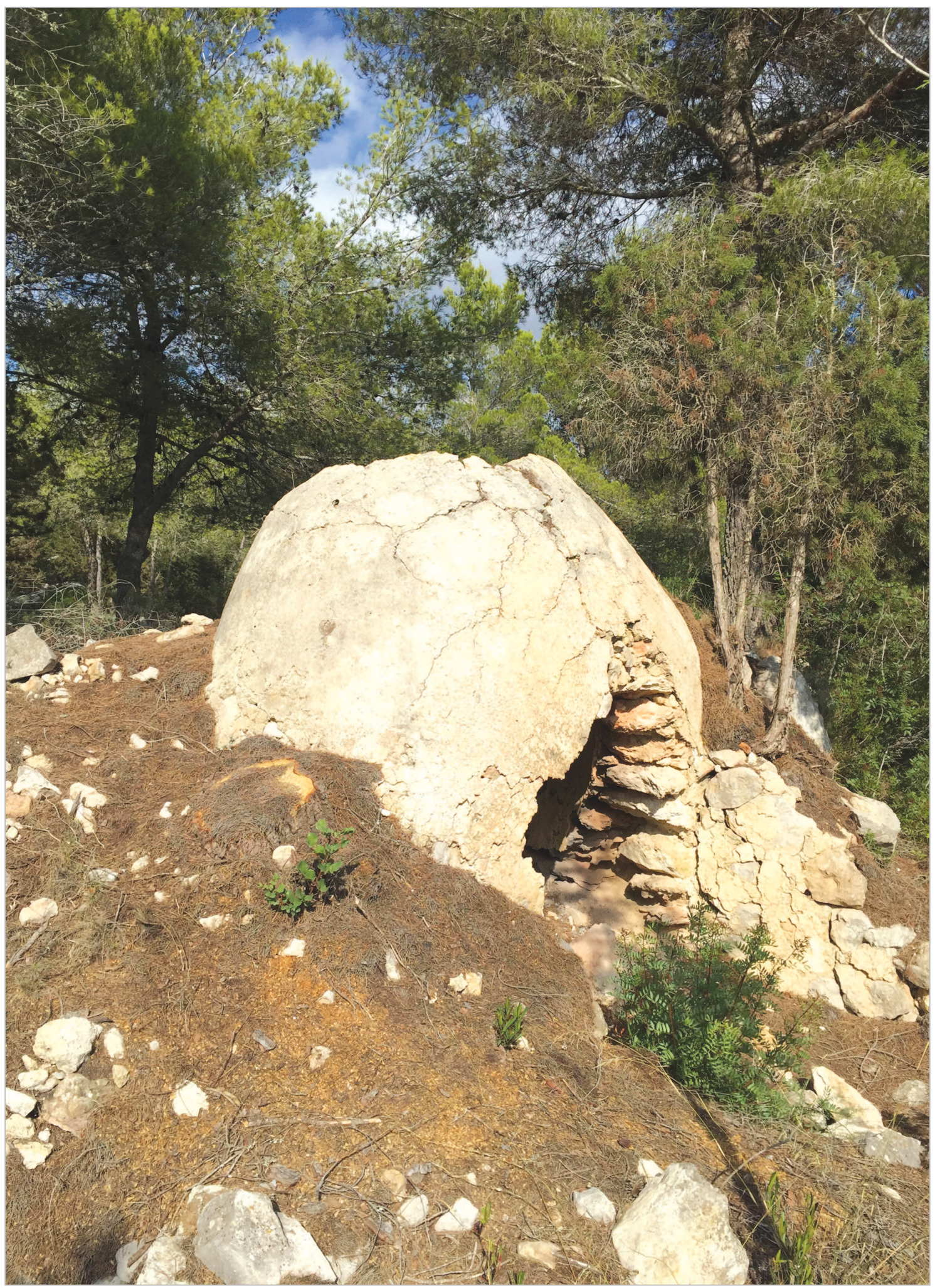

Figura 9. Vista lateral del horno de alquitrán (es forn) de Can Llucià des Camp Vell. El orificio frontal se corresponde con un derrumbe de la parte frontal, por encima de s'olla. 
ciantes locales, Can Cabrit y $\mathrm{Ca} \mathrm{Na}$ Cabeieta en el caso de la ciudad de Ibiza, que a su vez la enviaban a Barcelona. Este testimonio concuerda con la importancia de la resina en aquel momento, así como con la ausencia de referencias a la presencia de fábricas de transformación de resina en las Islas Baleares en aquellos años (Junta Intersindical de Resinas, 1950).

Los resultados obtenidos a partir de los informadores concuerdan en gran medida con la publicación de Guerau (1973), que es un completo artículo sobre la explotación de la resina y los hornos de alquitrán en la isla de Ibiza, desconocido paras los informadores. Este hecho da una mayor consistencia a las informaciones y también permite detectar algunas diferencias para las que sería de interés avanzar en futuras investigaciones sobre el tema. La más relevante es la relativa a la continuidad entre las operaciones de escimerolar y parar es pi. Para Marí (2018) eran dos operaciones consecutivas, sin solución de continuidad, mientras que para Guerau (1973) existía un periodo de reposo de tres a cuatro años entre ambas. Otra situación llamativa es la diversidad de cifras en relación con la duración del proceso de cocción. Las indicadas por los informadores resultan significativamente inferiores a las facilitadas por otros autores. Iturralde y Elorrieta (1914) indican una duración del fuego de dos días para hornos más o menos similares empleados en el aprovechamiento de residuos de las fábricas de resina. Guerau (1973) hace referencia a una duración del proceso de entre dos y cinco días. Otros autores hacen referencia a procesos de 2 a 4 días, variables en función de al menos la cantidad de madera cargada en el horno (Prados, ca 198-]; Fàbrega, 2006; Orduna, 2014) e incluso un periodo de 8 días para una primera carga (Fàbrega, 2006). Se considera que estas diferencias pueden responder a factores ya señalados, como son los relacionados con el tamaño del horno y la correspondiente carga de madera, o la temperatura en el momento del inicio de la cocción (menor duración para una segunda carga con el horno caliente); pero también puede ser muy relevante un tercer factor: las diferentes condiciones en el momento actual, con una previsible menor experiencia y pericia, así como sin una necesidad vital de obtención del producto.

Otras informaciones sí se mantienen en unos rangos similares. Es el caso de los turnos de extracción de madera teosa para un pino dado, para los que los diferentes informadores coinciden tanto en un valor común de dos años, como en la existencia de variantes de tres (Guerau, 1973) o de hasta cinco años en periodos anteriores, de menor intensidad de extracción (Marí, 2019).

En otros casos las diferencias existentes pueden servir para completar los conocimientos en situaciones particulares o en la recogida de la terminología empleada. De esta forma Guerau (1973) indica la existencia de algunos hornos en los que la abertura superior es central; también aporta otros términos para denominar algunas labores, como encanar es forn para el proceso del llenado del horno, la denominación de cova o creu de ses teies al hueco para evitar la obturación del rajador, o la denominación como sa corona y sa sola de los dos resaltes, superior e inferior, que enmarcan en el tronco del pino la zona de extracción.

La referencia a la posibilidad de obtención de pez a partir de la quema del alquitrán (Marí, 2019) coincide con el proceso indicado por otros autores (Iturralde y Elorrieta, 1914; Prados, ca 198-) y también con la acepción aportada en la Enci- 
clopèdia d'Eivissa i Formentera (Consell d'Eivissa, 2019), que define el encrità como un aceite espeso obtenido de destilar teas de pino, mientras que la pega es una sustancia muy viscosa que se obtiene de destilar el encrità de pino.

La observación de la evolución de los pinos, una vez realizada la operación de corta de la copa (escimerolar es pins), puede hacer pensar en un objetivo múltiple de la actividad: A corto plazo, la ya indicada obtención de madera teosa, de la que se podía obtener un mayor rendimiento en pez y con unas características especiales. A medio plazo, la posibilidad de obtención de piezas de madera con forma curva (Fig. 1), pues eran muy apreciadas en la construcción naval. Se ha consultado esta posibilidad a los informadores (Marí, 2019), sin que tengan constancia de tal pretensión.

Parece confirmarse el mantenimiento de la actividad hasta la década de los años treinta del siglo XX, con el mantenimiento de un cierto uso familiar hasta en torno el año 1960 (Guerau, 1973; Boned, 2018; Marí, 2018). Sería de interés conocer las causas del mantenimiento de esta actividad muchas décadas después de haber cesado en otros lugares de la península ibérica. Es un tema para el que es preciso un estudio más detallado y que puede ser complejo por la posible presencia de diversos factores interrelacionados. Para futuras investigaciones, se consideran posibles aspectos a tener en cuenta, entre otros:

1. El mantenimiento de una cierta abundancia de bosques en Ibiza y Formentera, frente a un más temprano y mayor deterioro de las masas arboladas en otras islas del archipiélago (Gil et al., 2002), que pudo permitir una continuidad en la producción ibicenca.

2. El posible mayor rendimiento en pez del empleo de ejemplares vivos de Pinus halepensis en lugar de madera seca o de madera de otras especies (Fàbrega, 2006).

3. La posible existencia de recursos humanos con los conocimientos técnicos precisos en la isla desde la antigüedad, que pudieron verse favorecidos por ser la isla de Ibiza un punto muy relevante de paso y descanso de los barcos desde los inicios de la navegación de las grandes civilizaciones en el Mediterráneo Occidental (Gil et al., 2002).

4. El mantenimiento de unas necesidades importantes de productos derivados de la resina para producciones básicas y principales de la isla, como el calafateado en la construcción y mantenimiento de la flota naval, o el empleo de tejidos y otros materiales impermeabilizados con alquitrán o sus derivados en la industria de la sal. Conviene destacar que la importancia de esta industria desde la Edad Antigua supuso la denominación de las Pityusas como las islas de la sal, así como que dicha actividad mantuvo su importancia (Vilà, 1953) durante todo el periodo histórico en el que se ha mantenido la producción local de alquitrán y pez. La condición insular ha podido tener una gran repercusión en el mantenimiento de la actividad. 


\section{Agradecimientos}

Agradecer a todas las personas que han facilitado a Vicent Agustí la información oral para la redacción de este artículo, especialmente: a Vicent Marí Serra 'Palermet', por mostrar su horno de alquitrán restaurado, explicar su historia y funcionamiento y por solucionar las dudas que han surgido; a Antoni Boned Boned "Basora", por los paseos por las sierras de Santa Agnés de Corona, mostrar el forn d'encrità de Ses Marrades de Corona y hacer a Vicent partícipe de la recuperación de esta tradición; a Antònia Bonet Bonet, "de Can Llucià des Camp Vell", por permitir ver el horno de alquitrán de su finca; a María Ribas Noguera "de Can Gibert", por dedicar su tiempo a explicar cómo era la recolección de resina; a Antoni Tur Riera "Sendic", por explicar el proceso de calafateo de las embarcaciones tradicionales ibicencas; a sus abuelas, Antònia Cardona Cardona y Maria Serra Torres, siempre fuente de conocimiento a través de sus palabras, sus recuerdos, historias y vivencias.

\section{Bibliografía}

Boned, A., 2019. Comunicación personal.

Bonet, A., 2018. Comunicación personal.

Cardona, A., 2019. Comunicación personal.

Consell d'Eivissa, 2019. Enciclopèdia d'Eivissa i Formentera. http://www.eeif.es/ Consulta: 15 de octubre de 2019

Fàbrega, A.; 2006. La pega vegetal. Producció i pluriactivitat pagesa. Estudis d'Historia Agraria $19 ; 69-104$.

Gil, L.; Valdés, C.M.; Díaz-Fernández, P.; 2002. La transformación histórica del paisaje forestal en las islas Baleares. Tercer Inventario Forestal Nacional 1997-2007. Ministerio de Medio Ambiente.

Guerau, C.; 1973. Los hornos de alquitrán, una explotación poco conocida de nuestros bosques. Revista Eivissa 3, 23-28.

Iturralde, J.; Elorrieta, O.; 1914. Estudio sobre la resinación de los montes españoles en sus aspectos botánicos, forestal, industrial y económico. Instituto de Ingenieros Civiles. Madrid.

Junta Intersindical de Resinas, 1950. Memoria del Plan Nacional de Resinas. Madrid.

Marí, V.; 2018. Comunicación personal.

Marí, V.; 2019. Comunicación personal.

Michavila, A.; 2010. Alquitraners dels nostres montes. Lo Senienc: memoria, natura y llengua 7, 11-16.

Orduna, P.; 2014. Aproximación etnohistórica al trabajo de la pez en las Bárdenas Reales (Navarra). Revista de Dialectología y Tradiciones Populares LXIX (2), 413-433. https://doi.org /10.3989/rdtp.2014.02.008

Prados, B.; ca 198-]. Las pegueras.

Real Academia Española, 2019. Diccionario de la lengua española, 23. ${ }^{a}$ ed., [versión 23.3 en línea]. https://dle.rae.es Consulta: 20 de junio de 2020.

Ribas, M.; 2018. Comunicación personal. 
Ribas, V.A.; 2019. Proyecto piloto de establecimiento de parcela de resinación de Pinus halepensis con objetivo múltiple en Sant Josep de Sa Talaia, Eivissa (Illes Balears). Trabajo Final de Grado. Escuela Técnica Superior de Ingeniería Agronómica y del Medio Natural. Universitat Politècnica de València. Valencia.

S.E.C.F., sin fecha. Glosario Técnico Forestal de la Sociedad Española de Ciencias Forestales. http://secforestales.org/diccionario_forestal_secf Consulta: 20 de junio de 2020.

Tur, A.; 2018. Comunicación personal.

Vilà, J.; 1953. Ibiza y Formentera, islas de la sal. Estudios Geográficos Instituto Juan Sebastián Elcano del CSIC XIV, 363-408. 
